EESTI NSV TEADUST̃E AKADEEMIA TOIMETISËD. 29, KÖIDË FOUSIKA * MATEMAATIKA, 1980, NR. 2

$\theta$

ИЗВЕСТИЯ АКАДЕМИИ НАУК ЭСТОНСКОП ССР. ТОМ 29 ФИЗИКА * МАТЕМАТИКА. 1980, № 2

Х. НЯРИПЯ

\title{
НЕКОТОРЫЕ НОВЫЕ ОЦЕНОЧНЫЕ ЗАДАЧИ К ЗАДАЧАМ ЦЕЛОЧИСЛЕННОГО ЛИНЕИНОГО ПРОГРАММИРОВАНИЯ
}

\author{
(Представил А. Хумал)
}

\section{1. Сравнение некоторых оценочных задач}

Рассмотрим задачу целочисленного линейного программирования (ЦЛП)

$$
Z_{0}=\min _{x \in X}\{c x \mid N x \leqslant b\}
$$

где $x-n$-мерный вектор-столбец, $c$ - неотрицательная $n$-мерная вектор-строка, $b$ - неотрицательный $m$-мерный вектор-столбец, $N=$ $=\left(a_{1}, \ldots, a_{n}\right)-(m \times n)$-матрица, а

$$
X=\left\{x \mid \sum_{j=1}^{n} \alpha_{j} x_{j} \equiv \beta, x \geqslant 0 \text { и целое }\right\}
$$

определяет бесконечную решетку целочисленных точек, удовлетворяющих системе линейных сравнений. Отметим, что любая задача ЦЛП, в случае ограниченности целевой функции соответствующей ей непрерывной задачи, приводима к виду (1) [ $\left.{ }^{1}\right]$ (с. $\left.378-388\right)$.

Известен целый ряд оценочных задач к задачам ЦЛП (см., напр., $\left.\left[{ }^{2}\right]\right)$, в том числе и задача групповой минимизации $\min \{c x \mid x \in X\}$ и задача

$$
Z_{1}=\max _{u \geqslant 0} \min _{x \in X}\{c x+u(N x-b)\},
$$

предложенная в $\left[^{3}\right]$. В настоящей работе изучаются еще две оценочные задачи

$$
Z_{2}=\max _{u \geqslant 0} \min _{x \in X}\{c x \mid u(N x-b) \leqslant 0\}
$$

и

$$
Z_{3}=\min _{z \geqslant 0} \max _{0 \leqslant u \leqslant e} \min _{x \in X}\{c x \mid z-c x+u(N x-b) \leqslant 0, c x \leqslant z\}
$$

где $e$ - вектор, состоящий только из единиц. Строится также алгоритм решения задачи (4) (алгоритм решения задачи (3) аналогичен и поэтому опускается). 
Пусть $x_{0}^{*}$ - оптимальное решение задачи (1), $\left(u_{1}^{*}, x_{1}^{*}\right)$ и $\left(u_{2}{ }^{*}, x_{2}{ }^{*}\right)$ - оптимальные решения задач (2) и (3), а $\left(z_{3}^{*}, u_{3}^{*}, x_{3}^{*}\right)$ оптимальное решение задачи (4).

Т е о рем а 1. Справедливы неравенства

$$
Z_{1} \leqslant Z_{2} \leqslant Z_{3} \leqslant Z_{0}
$$

Док а 3 а те льст в о. Так как $c x_{0}{ }^{*}=Z_{0}$ и для любого $u \geqslant 0$ имеем $u\left(N x_{0} *-b\right) \leqslant 0$, то

$$
Z_{3} \leqslant \max _{0 \leqslant u \leqslant e} \min _{x \in X}\left\{c x \mid Z_{0}-c x+u(N x-b) \leqslant 0, c x \leqslant Z_{0}\right\} \leqslant Z_{0} .
$$

Из оптимальности $z_{3}{ }^{*}$ следует, что при любом $0 \leqslant u \leqslant e$

$$
\min _{x \in X}\left\{c x \mid z_{3}^{*}-c x+u(N x-b) \leqslant 0, c x \leqslant z_{3}^{*}\right\} \leqslant Z_{3}
$$

а так как при ослаблении ограничений минимум не может увеличиваться, то и

$$
\min _{x \in X}\{c x \mid u(N x-b) \leqslant 0\} \leqslant Z_{3} .
$$

Последнее неравенство остается справедливым для всех $u \geqslant 0$, следовательно,

$$
Z_{2}=\max _{0 \leqslant u} \min _{x \in X}\{c x \mid u(N x-b) \leqslant 0\} \leqslant Z_{3} .
$$

Неравенство $Z_{1} \leqslant Z_{2}$ доказывается аналогично предыдущему, и тем самым теорема доказана.

\section{2. Исследование новой оценочной задачи и одна ее модификация}

Изложим некоторые свойства оптимальных решений задачи (4).

Т е о р е а 2. Если задача (4) имеет конечное оптимальное решение, то $Z_{3}=z_{3}^{*}$.

Доказательство. В силу ограничения $c x \leqslant z$ имеем $Z_{3}=$ $=c x_{3}^{*} \leqslant z_{3}^{*}$. С другой стороны,

$$
\begin{gathered}
Z_{3}=\max _{0 \leqslant u \leqslant e} \min _{x \in X}\left\{c x \mid z_{3}^{*}-c x+u(N x-b) \leqslant 0, c x \leqslant z_{3}^{*}\right\} \geqslant \\
\geqslant \min _{x \in X}\left\{c x \mid z_{3}^{*}-c x \leqslant 0, c x \leqslant z_{3}^{*}\right\} \geqslant z_{3}^{*} .
\end{gathered}
$$

Теорема доказана.

Введем следующие обозначения:

$$
\begin{aligned}
& L(z, u)=\min _{x \in X}\{c x \mid z-c x+u(N x-b) \leqslant 0\}, \\
& L(z)=\max _{0 \leqslant u \leqslant e} L(z, u) .
\end{aligned}
$$

Т еорем а 3. Если $\bar{z} \leqslant z_{3}{ }^{*}$, то 1) при любом $0 \leqslant \bar{u} \leqslant е$ выполняется неравенство $L(\bar{z}, \bar{u}) \leqslant Z_{3} u$ 2) из равенства $L(\bar{z})=\bar{z}$ следует равенство $\bar{z}=z_{3}^{*}$.

Доказ а тельств о. Оценим $Z_{3}$ снизу: 


$$
\begin{aligned}
Z_{3} & =\max _{0 \leqslant u \leqslant e x \in X}\left\{c x \mid z_{3}^{*}-c z+u(N x-b) \leqslant 0, c x \leqslant z_{3}^{*}\right\} \geqslant \\
& \geqslant \min _{x \in X}\left\{c x \mid z_{3}^{*}-c x+\bar{u}(N x-b) \leqslant 0, c x \leqslant z_{3}^{*}\right\} \geqslant \\
& \geqslant \min _{x \in X}\left\{c x \mid z_{3}^{*}-c x+\bar{u}(N x-b) \leqslant 0\right\}=L\left(z_{3}^{*}, \bar{u}\right) \geqslant L(\bar{z}, \bar{u}) .
\end{aligned}
$$

Докажем теперь второе утверждение теоремы. Если $L(\bar{z})=\bar{z}$, то для любого $0 \leqslant u \leqslant e$ существует $x(u) \in X$ такое, что $\bar{z}-c x(u)+$ $+u(N x(u)-b) \leqslant 0$ и $c x(u) \leqslant \bar{z}$. Следовательно,

$$
\begin{gathered}
\bar{z}=\max _{0 \leqslant u \leqslant e} \min _{x \in X}\{c x \mid \bar{z}-c x+u(N x-b) \leqslant 0, c x \leqslant \bar{z}\} \geqslant \\
\geqslant \min _{z \geqslant 0} \max _{0 \leqslant u \leqslant e} \min _{x \in X}\{c x \mid z-c x+u(N x-b) \leqslant 0, c x \leqslant z\}=Z_{3}
\end{gathered}
$$

и, в силу предположения $\bar{z} \leqslant z_{3}^{*}=Z_{3}, \bar{z}=z_{3}{ }^{*}$. Теорема доказана.

Пусть $J^{0}=\left\{j \mid c_{j}=0\right\}, d_{j}=\min \left\{t \mid t \alpha_{j} \equiv 0, t \geqslant 1\right\}-$ порядок циклической группы, генерированной вектором $\alpha_{i}, X_{1}=\left\{x \in X \mid x_{j} \leqslant d_{j}-1\right.$, $\left.j \in J^{0}\right\}$ и $L_{1}(z, u)=\min _{x \in X_{1}}\{c x \mid z-c x+u(N x-b) \leqslant 0\}$.

Т е о рем а 4. Нмеет место равенство

$$
L(z)=\max _{0 \leqslant u \leqslant e}\left\{L_{1}(z, u) \mid u a_{j} \geqslant 0, j \in J^{0}\right\} .
$$

Дока за тельство. Сначала докажем, что $L(z)=\max _{0 \leqslant u \leqslant e}\{L(z, u) \mid$ $\left.\mid u a_{j} \geqslant 0, j \in J^{0}\right\} \equiv L_{1}(z)$. По определению $L_{1}(z) \leqslant L(z)$. Пусть $L(z)=$ $=L(z, \bar{u})$. Если $\bar{u} a_{j} \geqslant 0$ для всех $\ddot{j} \in J^{0}$, то $L_{1}(z) \geqslant L(z, \bar{u})=L(z)$ и, следовательно, $L_{1}(z)=L(z)$. Предположим теперь, что при некотором $k \in J^{0}$ имеем $\bar{u} a_{k}<0$. Обозначим оптимальное решение задачи $\min \{c x \mid x \in X\}$ через $\bar{x}$ и определим $\bar{x}(t)=\bar{x}+t d_{k} e_{k}$, где $e_{k}-k$-й единичный вектор. Тогда при любом целочисленном неотрицательном $t$ выполняются условия $\bar{x}(t) \in X$ и $c \bar{x}(t)=c \bar{x}$. Так как $\bar{u} a_{k}<0$, то существует неотрицательное целочисленное $\bar{t}$, для которого $z-c \bar{x}(\bar{t})+$ $+u(N \bar{x}(\bar{t})-b) \leqslant 0 . \quad$ Следовательно, $L(z)=L(z, \bar{u})=c \bar{x} \leqslant$ $\leqslant \min \{c x \mid z-c x \leqslant 0, x \in X\}=L(z, 0) \leqslant L_{1}(z)$ и снова $L(z)=L_{1}(z)$.

Теперь докажем, что если для всех $j \in J^{0}$ верно $u a_{j} \geqslant 0$, то $L_{1}(z, u)=$ $=L(z, u)$. Пусть $x(z, u)$ - оптимальное решение задачи (5), т. е. $c x(z, u)=L(z, u)$. Для каждого $j \in J^{0}$ найдется целочисленное $t_{j} \geqslant 0$ такое, что $0 \leqslant x_{j}(z, u)-t_{j} d_{j} e_{j} \leqslant d_{j}-1$. Легко проверить, что вектор $\bar{x}(z, u)=x(z, u)-\sum_{j \in J^{v}} t_{j} d_{j} e_{j} \quad$ удовлетворяет следующим условиям: $\bar{x}(z, u) \in X_{1}, c \bar{x}(z, u)=c x(z, u) \quad$ и $\quad z-c \bar{x}(z, u)+u(N \bar{x}(z, u)-b) \leqslant 0$. Тогда $L_{1}(z, u) \leqslant c \bar{x}(z, u)=c x(z, u)=L(z, u)$, но так как $X_{1} \subset X$, то справедливо неравенство $L_{1}(z, u) \geqslant L(z, u)$ и тем самым $L_{1}(z, u)=$ $L(z, u)$.

Утверждение теоремы следует непосредственно из доказанных выше двух утверждений.

Доказанная теорема позволяет вместо задачи (4) решать ее модификацию, в которой $X$ заменено на $X_{1}$. Пересечение $X_{1}$ на любое множество уровня целевой функции $c x$ является конечным множеством, что очень важно для построения конечных алгоритмов. 


\section{3. Решение оценочной задачи}

Опишем алгоритм решения задачи (4).

Шаг 0 . Присвоить параметрам начальные значения: $k:=1, z^{1}:=0$ и $u^{1}:=0$.

UІ а г 1. Решить задачу

$$
\min _{x \in X_{1}}\left\{c x \mid z^{k}-c x+u^{k}(N x-b) \leqslant 0\right\},
$$

которая является задачей групповой минимизации с одним дополнительным линейным ограничением. *

Если задача (6) не разрешима, то у задачи (1) отсутствуют допустимые решения и работа алгоритма завершена. В противном случае взять $z^{k+1}:=\max \left\{z^{k}, c x^{h}\right\}$, где $x^{k}$ - оптимальное решение задачи (6), и перейти к шагу 2.

Шаг 2. Найти оптимальное решение $\left(\sigma^{k}, u^{k+1}\right)$ задачи

$$
\begin{aligned}
& \quad \max \sigma \\
& \sigma \leqslant u\left(N x^{t}-b\right)+z^{h+1}-c x^{t}, t=1, \ldots, k, \\
& 0 \leqslant u a_{j}, j \in J^{0}, \\
& 0 \leqslant u \leqslant e .
\end{aligned}
$$

Задача (7) является задачей линейного программирования с ограниченной целевой функцией и всегда имеет оптимальное решение.

Если $\sigma^{k}=0$, то $Z_{3}=z_{3}{ }^{*}=z^{k+1}$ и оптимальным решением задачи (4) является $\left(z^{k+1}, u^{t}, x^{t}\right)$ при любом $t \in I=\left\{i \mid c x^{i}=z^{k+1}, i=1, \ldots, k\right\}$. Работа алгоритма завершена. В противном случае $\sigma^{k}>0$, ибо $\sigma=0$, $u=0$ является допустимым решением любой задачи (7). Взять $k$ равным $k+1$ и перейти к шагу 1 .

\section{4. Обоснование алгоритма}

Покажем, что для всех $k$ верно $z^{k} \leqslant z_{3}^{*}$. Очевидно, $z^{1}=0 \leqslant z_{3}{ }^{*}$. Пусть, далее, $z^{k} \leqslant z_{3}{ }^{*}$ и покажем, что тогда и $z^{h+1} \leqslant z_{3}{ }^{*}$. По определению $z^{k+1}=\max \left\{z^{k}, c x^{k}\right\}$. Если $z^{k+1}=z^{k}$, утверждение справедливо по предположению $z^{k} \leqslant z_{3}{ }^{*}$, а если $z^{k+1}=c x^{k}$, 一 по теоремам 4 и $3: z^{k+1}=$ $=c x^{k}=L_{1}\left(z^{k}, u^{k}\right)=L\left(z^{k}, u^{k}\right) \leqslant z_{3}{ }^{*}$.

Докажем теперь, что если задача (1) имеет допустимые решения, то они имеются и у всех задач $(6)$. Пусть $\bar{x}-$ некоторое допустимое решение задачи (1). Тоғда $N \bar{x}-b \leqslant 0$, а также для всех $u \geqslant 0$ имеем $u(N \bar{x}-b) \leqslant 0$. Так как $c \bar{x} \geqslant Z_{0} \geqslant Z_{3}=z_{3}{ }^{*} \geqslant z^{k}$ при любом $k$, то $z^{k}-c \bar{x}+u^{k}(N \bar{x}-b) \leqslant 0$ и, следовательно, $L\left(z^{k}, u^{k}\right) \leqslant c \bar{x}$. По теореме 4 $L_{1}\left(z^{k}, u^{k}\right)=L\left(z^{k}, u^{k}\right) \leqslant c \bar{x}$, что возможно только тогда, когда соответствующая задача (6) имеет по меньшей мере одно допустимое решение.

Остается доказать, что из $\sigma^{k}=0$. следует $z^{k+1}=z_{3}^{*}$. Если $\sigma^{k}=0$, то для каждого $u \in U=\left\{u \mid 0 \leqslant u \leqslant e, u a_{j} \geqslant 0, j \in J^{0}\right\}$ существует $x(u) \in\left\{x^{t} \mid t=1, \ldots, k\right\} \quad$ такое, что $\quad c x(u) \leqslant z^{k+1}=\max _{t=1, \ldots, k} c x^{t} \quad$ и $z^{k+1}-c x(u)+u(N x(u)-b) \leqslant 0$. Следовательно, для всех $u \in U$ верно $L_{1}\left(z^{k+1}, u\right) \leqslant z^{k+1}, \quad$ но, с другой стороны, $L_{1}\left(z^{k+1}, 0\right)=z^{k+1}$ и тем самым $\max L_{1}\left(z^{k+1}, u\right)=z^{k+1}$. Тогда по теореме $4 L\left(z^{k+1}\right)=z^{k+1}$ и по теореме 3 $u \in U$ $z^{k+1}=z_{3}^{*}=Z_{3}$.

* Методы решения таких задач будут опубликованы отдельно. 


\section{5. Конечность алгоритма и некоторые замечания}

Покажем, что если задача (1) имеет допустимое (тогда и оптимальное) решение, то описанный выше алгоритм завершается через конечное число итераций.

При сделанном предположении $z_{3}{ }^{*}=Z_{3} \leqslant Z_{0}<\infty$, а по определению алгоритма для всех $k$ имеем $c x^{k} \leqslant z^{k+1} \leqslant z_{3}{ }^{*}$ и $x^{i} \neq x^{j}$, если $i \neq j$ (это гарантируется определением $u^{k}$ в задаче (7)). Конечность алго-

ритма вытекает из конечности множества $X\left(z_{3}{ }^{*}\right)=\left\{x \in X_{1} \mid c x \leqslant z_{3}{ }^{*}\right\}$.
Сделаем еще некоторые замечания:

1. Если на некоторой итерации $N x^{h} \leqslant b$, то $x^{k}$ является оптимальным решением задачи (1).

2. Если задача (1) не имеет допустимых решений, описанный алгоритм может оказаться бесконечным, но тогда неограниченно увеличивается и нижняя граница $z^{k}$ оптимального значения целевой функции задачи (1). 3. На втором шаге алгоритма можно ограничиться любым решением $\left(\bar{\sigma}^{k}, \bar{u}^{k+1}\right)$ задачи $(7)$, при котором $\bar{\sigma}^{k}>0$.

4. На каждой итерации алгоритма можно вместо $u^{h}$ пользоваться вектором $\bar{u}^{k}=\lambda_{k} u^{k}$ при любом $\lambda_{k} \in(0,1]$. Далее можно описать правила определения $\lambda_{k}$ такого, что использование на шаге $1 \bar{u}^{k}$ вместо $u^{k}$ приведет к выполнению условия $z^{k+1} \geqslant z^{k}$. При этом уменьшится также число ограничений в задачах (7).

Примечание при корректуре. Во время набора настоящей статьи автору стали известны результаты, изложенные в $\left[{ }^{4}\right]$, где более подробно изучены свойства задач $(2)$ и (3), а также предложен алгоритм решения задачи (3).

\section{Л ИТЕРА Т У РА}

1. Х у Т., Целочисленное программирование и потоки в сетях, М., «Мир», 1974.

2. Л ебе дев C. C., Экономика и матем. методы, 10, вып. 3, 592-610 (1974)
3. Fis her, M. L., S ha p ir o, J. F., SIAM J. Appl. Math., 27, № 1, 31-52 (1974).
4. Ka rwa n, M. M. 4. Ka r w a n, M. M., Rard i n, R. L., Math. Programming, 17, № $3,320-334$ (Nov.
1979).

Тартуский государственный университет

Поступила в редакцию $11 / \mathrm{XI} 1979$

\section{H. NARIPA}

\section{UUTE HINNANGUTE LEIDMISEST TÄISARVULISE LINEAARSE PLANEERIMISOLESANDE SIHIFUNKTSIOONILE}

Täisarvulist lineaarset planeerimisülesannet on vaadeldud kujul

$$
Z_{0}=\min _{x \in X}\{c x \mid N x \leqslant b\}
$$

kus $X=\left\{x \mid \sum_{j=1}^{n} \alpha_{j} x_{j} \equiv \beta, \quad x \geqslant 0\right.$ ja täisarv $\}$ määrab lineaarset kongruentside süsteemi rahuldavate täisarvuliste mittenegatiivsete punktide lõpmatu hulga. Selle ülesande sihifunktsiooni optimaalse väärtuse $Z_{0}$ alumise tõkke $Z$ leidmiseks on konstrueeritud üles-
anne

$$
Z=\min _{z \geqslant 0} \max _{0 \leqslant u \leqslant e} \min _{x \in X}\{c x \mid z-c x+u(N x-b) \leqslant 0, c x \leqslant z\}
$$

ja esitatud lõplik iteratiivne algoritm selle lahendamiseks. Algoritmi igal sammul tuleb lahendada üks lineaarse lisakitsendusega rühmaminimeerimisülesanne ja üks linearne
planeerimisülesanne. 


\section{H. NARIPA}

\section{ON FINDING ESTIMATIONS FOR THE OBJECTIVE FUNCTION OF A LINEAR INTEGER PROGRAMMING PROBLEM}

A linear integer programming (IP) problem is considered in the form:

$$
Z_{0}=\min _{x \in X}\{c x \mid N x \leqslant b\}
$$

where $X=\left\{x \mid \sum_{j=1}^{n} \alpha_{j} x_{j} \equiv \beta, x \geqslant 0, x\right.$-integer $\}$ determines an infinite set of non-negative integer points satisfying the system of linear congruences. One well-known way to find a lower bound for such a problem is to solve the corresponding group minimization problem $\min \{c x \mid x \in X\}$. To obtain a stronger bound, many authors have resorted to various approaches, using the Lagrange multipliers theory for integer problems. Int this paper a synthesis of two such approaches is considered: one of these has been given by Lebedew and another by Fisher and Shapiro. The lower bound $Z$ to the given IP problem is searched in the form:

$$
Z=\min _{z \geqslant 0} \max _{0 \leqslant u \leqslant e} \min _{x \in X}\{c x \mid z-c x+u(N x-b) \leqslant 0, c x \leqslant z\} .
$$

It is shown that the new bound is stronger than that of Fisher and Shapiro. Also, a finite iterative method for determining $Z$ is described, at each step of which a group minimization problem with an additional linear constraint and a linear programming problem have to be solved. In addition to a relatively strong bound, this method also generates a set of integer points in the corner polyhedron corresponding to the IP problem. Each of these points satisfies a surrogate constraint and therefore in many cases it may prove possible to find exact solution to the IP problem. 